Original

\title{
Tratamiento laparoscópico de la ureterolitiasis: nuestra experiencia
}

\author{
Danilo Borges Matias, Ricardo Gonçalves Alvim, Mateus Ribas, Bruno Pierote de Oliveira, \\ Otto H. Torres Chaves.
}

Servicio de Urología. Hospital Universitario São José, Belo Horizonte, Minas Gerais, Brasil

\begin{abstract}
Resumen
Objetivo: Analizar detalles técnicos y resultados del tratamiento laparoscópico de la ureterolitiasis. Pacientes y métodos: En el periodo de enero de 2005 a agosto de 2008, han sido realizadas 22 ureterolitotomías laparoscópicas (UL) en nuestro servicio; dos (9\%) han sido por acceso retroperitoneal y 20 (91\%) por acceso transperitoneal. La media de tamaño del cálculo fue de $15 \mathrm{~mm}$, variando de 8 a $23 \mathrm{~mm}$, y los cálculos estaban en uréter proximal en 18 pacientes $(81,8 \%)$, uréter mediano en 4 pacientes $(18,2 \%)$ y ninguno en uréter distal. La mayoría de los cálculos eran obstructivos hacía más de dos meses. Ocho pacientes han sido sometidos a sesiones previas de litotricia extracorpórea por ondas de choque y 2 a litotricia extracorpórea con ureterolitotricia en el intento de tratamiento de los cálculos. Doce pacientes han tenido ureterolitotomía laparoscópica como indicativo primario de tratamiento.

Resultados: La ureterolitotomía laparoscópica alcanzó éxito en 20 pacientes (90,9\%), ocurriendo fallos al inicio de nuestra experiencia. La media de tiempo operatorio fue de 145 minutos, variando de 70 a 240 . Fue utilizado catéter doble j en 3 casos, siendo 2 en el preoperatorio y 1 en el postoperatorio. La sutura ureteral ha sido realizada con hilo absorbible 4.0, con puntos separados en todos los pacientes. La media global de permanencia hospitalaria fue de 3,3 días y la de tiempo de permanencia del dreno fue de 7,2 días. La tasa global de complicaciones fue el 13,6\% y la de pacientes sin cálculo residual fue el $91 \%$, habiendo litiasis residual en 2 pacientes.

Conclusiones: El tratamiento laparoscópico de la litiasis ureteral, además de mínimamente invasivo, es viable, seguro y bastante eficaz. Debe de ser considerado como un procedimiento de salvamento para la litotricia extracorpórea por ondas de choque y ureterolitotricia. Sin embargo, en casos en los que hay cálculos obstructivos desde hace mucho tiempo, en pacientes seleccionados y, especialmente, en aquellos con riñón único, puede ser considerado como tratamiento de primera línea. En cuanto a la vía de acceso; si retroperitoneal o transperitoneal; todavía no se puede afirmar cual sería la mejor, y la opción entre una u otra debe de ser realizada de acuerdo con la preferencia y experiencia del cirujano.
\end{abstract}

Palabras clave: Laparoscopia. Litiasis ureteral. Ureterolitotomía. Extracción de cálculo. Retroperitoneoscopia.

\section{Laparoscopic treatment of ureterolithiasis: our experience}

\section{Abstract}

Objective: Analyze technical details and results of laparoscopic treatment of ureterolithiasis.

Materials and methods: Between January 2005 and August 2008 it was performed laparoscopic ureterolithotomy in 22 patients in our service. Two (9\%) by retroperitoneal access and $20(91 \%)$ by transperitoneal access. Mean size of the calculi was $15 \mathrm{~mm}$. with a variation from $8 \mathrm{~mm}$ to $23 \mathrm{~mm}$. The calculus were in the upper tract in 18 patients $(81,8 \%)$ and in mid ureter in 4 patients $(18,2)$, any of the calculus were in lower ureter. Most of calculi were obstructive stones for more than 2 months. Eight patients were submited a previous section of extracorporeal shockwave lithotripsy and 2 by extracorporeal shockwave lithotripsy plus ureterolithotripsy as a calculi treatment attempt. Twelve patients had a laparoscopic ureterolithotomy as a primary indication for treatment.

Results: Laparoscopic ureterolithotomy was successful for 20 patients (90,9\%), and the fails occurred in the beginning of our experiment. Mean operative time was 145 minutes with range from 70 to 240 minutes. The indwelling ureteral stent was used in 03 cases; 02 preoperative, and 01 postoperative. The ureteral suture was performed with absorbable 4.0 separated stitches in all patients. The global average of permanence in hospital was 3,3 days and the drain permanence was 7,2 days. The global complication rate was $13,6 \%$. The global rate of stone free was $91 \%$, and there were residual stone in 2 patients.

Conclusions: The laparoscopic treatment of ureterolithiasis besides minimally invasive, it is viable, secure and very effective. It should have taken into consideration as a procedure to extracorporeal shockwave lithotripsy and ureteroscopy. But, in cases where there are obstructive stones for a long time, in selected patients and, in special, in patients with just one kidney, it can be considered as a first line treatment. About the access, if retroperitoneal or transperitoneal; it is not still possible to affirm which would be the best one, the option must take into account the surgeon preference and experience.

Keywords: Laparoscopy. Ureteral calculi. Ureterolithotomy. Stone extraction. Retroperitoneoscopy. 
$\mathrm{E}$ los últimos años hubo un gran desarrollo en técnicas mínimamente invasivas en el tratamiento de litiasis urinaria asociada a una tecnología punta, como la litotricia extracorpórea por ondas de choque (LEOC), el láser y la ureterorrenoscopia flexible, con alta eficacia y baja morbilidad. Mientras tanto, los pacientes con grandes cálculos obstructivos, especialmente aquellos con uréter proximal, o los casos en que hay anomalías ureterales, representan gran desafío desde el punto de vista terapéutico, siendo frecuentemente necesarias múltiples intervenciones. Hay que considerar, también, los casos de litiasis en pacientes portadores de riñón único. En esos casos, la cirugía abierta, convencional, todavía es aplicable.

Desde la descripción inicial hecha por Wickham ${ }^{1}$ en 1979 y su difusión, especialmente vía retroperitoneal, descripta por Gaur ${ }^{2}$ a partir de 1993, la ureterolitotomía laparoscópica (UL) sigue ganando espacio, sustituyendo la técnica abierta, especialmente en centros en los que no se tiene acceso universal a equipamientos de endourología.

El presente estudio presenta nuestra experiencia y análisis técnico del empleo de la ureterolitotomía en un grupo de pacientes seleccionados.

\section{PACIENTES Y MÉTODOS}

En el periodo de enero de 2005 a agosto de 2008, 22 pacientes han sido sometidos a UL para tratamiento de cálculos ureterales en nuestro servicio, siendo 2 vía retroperitoneal y 20 (91\%), vía transperitoneal. La relación hombre/mujer fue de 2:1. Los cálculos eran obstructivos crónicamente en todos los pacientes, con la media de 2 meses, variando de uno a cuatro meses. El abordaje laparoscópico ha sido utilizado como método de salvamento para LEOC o ureteroscopia en 10 pacientes $(45,5 \%)$. La UL fue indicada como terapia primaria en 12 pacientes $(54,5 \%)$.

En todos los pacientes, el diagnóstico ha sido confirmado con exámenes de imagen (rayos X simples del abdomen, ultrasonografía y urograma intevenoso o tomografia axial computerizada del abdomen). Han sido encontrados cálculos a la derecha en 15 pacientes $(68,2 \%)$ y a la izquierda en siete $(31,8 \%)$. La media (variación) de edad de los pacientes fue de 39 años (23-58). El tamaño medio (variación) de los cálculos fue de $1,3 \mathrm{~cm}(0,8-2,3)$, estando $18(81,8 \%)$ ubicados en el uréter superior y $4(18,2 \%)$ en el uréter medio. En todos los pacientes fue realizado con anestesia general con intubación orotraqueal.

\section{Técnica Operatoria}

En cuanto a la vía de acceso, dos pacientes han sido sometidos a retroperitoneoscopia y veinte, vía transperitoneal. Con el paciente en decúbito lateral a $90^{\circ}$, en la vía transperitoneal, el neumoperitoneo fue instaurado por vía abierta, transumbilical, y puesto un trócar de Hasson (con una sutura en bolsa para evitar el escapamiento de gas) en todos los pacientes. Los otros trócares han sido posicionados en la línea hemiclavicular ipsilateral. Cuando fue necesaria una mayor exposición, un cuarto trócar de $5 \mathrm{~mm}$ era posicionado en la línea axilar media ipsilateral. Han sido realizados tres trócares en $6(27,3 \%)$ y cuatro en 16 pacientes $(72,7 \%)$.

Cuando se utilizaba la vía retroperitoneal, el acceso era obtenido con la técnica abierta, a través de una incisión de $15 \mathrm{~mm}$ en la extremidad de la $12^{\mathrm{a}}$ costilla, divulsión muscular hasta la aponeurosis del músculo transverso, que creaba el espacio con disección digital, seguida de la introducción de un balón, conforme está descrito por Gaur ${ }^{3}$. Después de pasados 2 trócares adicionales bajo visión -siendo uno de $5 \mathrm{~mm}$ en línea hemiclavicular, sobre la crista ilíaca y caudalmente al portal de la óptica, y otro de $10 \mathrm{~mm}$ en la línea axilar posterior- es compuesto un triangulo.

Se ha realizado incisión en el uréter con bisturí intracorpóreo en 14 pacientes $(63,6 \%)$ y con endotijeras en siete $(36,4 \%)$. El cálculo ha sido extraído con una pinza de agarre atrumática en 17 pacientes y con compresión del uréter en tres. La extracción del cálculo del abdomen ha sido hecha con un dedo de guante estéril en todos los pacientes. La sutura ureteral ha sido realizada con hilo absorbible 4.0, con puntos separados en todos los pacientes. Hubo implante de catéter ureteral (doble J) en 3 pacientes.

Un dreno de Penrose era posicionado en el espacio retroperitoneal y exteriorizado por uno de los portales, siendo extraído cuando el drenaje era mínimo. El catéter de doble $\mathrm{J}$ ha sido extraído tras 4 a 6 semanas después del examen radiológico.

\section{RESULTADOS}

La UL obtuvo éxito en 20 pacientes, habiendo fallado en 2, a causa de dificultades técnicas. El tiempo quirúrgico mediano (variación) fue de $132 \mathrm{~min}$ (70240). El tiempo de permanencia hospitalaria medio (variación) fue de 3,3 días (02-07), con uso de dreno con un promedio de 7,2 días, variando de 02 a 28 días. Dieciocho pacientes $(81,8 \%)$ han recibido alta hospitalaria con dreno, que era retirado en la primera visita postoperatoria. La abertura del uréter con bisturí o endotijeras no ha demostrado diferencia significativa en la tasas de drenaje postoperatoria. 
Ocurrió litiasis residual en 2 pacientes al inicio de nuestra serie, a causa de la migración del cálculo proximalmente y de la imposibilidad de localizarlo después.

La tasa global de complicación fue de 13,6\%.

\section{DISCUSIÓN}

No se puede negar que el advenimiento de nuevas técnicas mínimamente invasivas para el abordaje de la urolitiasis, como LEOC, ureterorrenoscopia flexible, cirugía percutánea y uso de láser, ha traído una fuerte disminución de la morbilidad ${ }^{3}$. Sin embargo, aún con todo ese desarrollo, algunos pacientes todavía tendrán indicación de cirugía abier$\mathrm{ta}^{4}$. La principal indicación para la UL es como un procedimiento de salvamento para LEOC o para fallo en la ureteroscopia ${ }^{5}$. En nuestra serie, hubo un número significativo de UL como indicación primera de tratamiento, por obstrucción prolongada de los cálculos, deseo de los pacientes de tener su enfermedad solucionada en un único abordaje o, incluso, por falta de acceso a otras modalidades de terapia mínimamente invasiva. Hay que resaltar que, en los países en desarrollo, aunque con necesidad de anestesia general, UL es un procedimiento más barato que LEOC o ureteroscopia ${ }^{5}$. La gran mayoría de los hospitales en nuestro país ya dispone de material de laparoscopia para tratamiento de la colecistopatía calculosa. La UL tiene, además, como ventajas la posibilidad de tratamiento de patologías asociadas (como la estenosis de la unión ureteropélvica) y la retirada del cálculo intacto, disminuyendo, de esa manera, el riesgo de litiasis residual.

UL es considerado un procedimiento urológico laparoscópico simple ${ }^{5}$, pero la identificación del uréter a veces puede ser difícil, tardía y frustrante, especialmente en su diferenciación con la vena gonadal. El uso del catéter ureteral (doble J) sigue siendo un tema controvertido ${ }^{6}$. Aunque haga más fácil la identificación del uréter en la disección, algunas veces son perdidos parámetros en la identificación del lugar exacto del cálculo, especialmente en un uréter distendido o espesado ${ }^{7}$.

Aunque la ureterolitiasis sea una patología común, existen pocos relatos de tratamiento laparoscópico de la misma en la Literatura. Algunos autores defienden el abordaje transperitoneal para tratamiento de los cálculos ureterales ${ }^{5-8}$, utilizando como principal argumento el amplio espacio de trabajo, especialmente para el tiempo quirúrgico de la reconstrucción. La corriente defensora del abordaje retroperitoneal tiene como principal difusor al Dr.
Gaur $^{2,4}$ y alega a su favor la reproducción de la técnica abierta original y la ausencia de contacto de un posible extravasamiento urinario con la cavidad peritoneal y sus consecuencias. La principal desventaja del acceso retroperitoneoscópico es el pequeño espacio para reconstrucción de la vía urinaria ${ }^{9}$.

En los casos con alteraciones anatómicas del tracto urinario, la laparoscopia representa una alternativa segura y eficaz para los procedimientos endoscópicos que suelen ser más difíciles y arriesgados en estas condiciones.

\section{CONCLUSIÓN}

La laparoscopia es un método que reproduce los pasos de la cirugía abierta, convencional, y puede ser indicada como alternativa en casos de fallo de otros métodos mínimamente invasivos. Mientras tanto, en casos en que el riesgo de fallo de tales métodos es considerado alto, como alteraciones anatómicas, cálculos voluminosos e impactados en uréter proximal, la laparoscopia puede ser indicada como terapia de primera línea ${ }^{4}$.

\section{REFERENCIAS}

1. Wickham JEA. The Surgical Treatment of Urinary Lithiasis. In Wickham JEA (ed), Urinary Calculus Disease. New York, Churchill Livingstone. 1979;145-98.

2. Gaur DD. Retroperitoneal laparoscopic ureterolithotomy. World J Urol. 1993;11(3):175-177.

3. Micali S, Moore RG, Averch TD, Adams JB, Kavoussi LR. The role of laparoscopy in the treatment of renal and ureteral calculi. J Urol. 1997:157(2):463-466.

4. Assimos DG, Boyce WH, Harrison LH, McCullough DL, Kroovand RL, Sweat KR The role of open stone surgery since extracorporeal shock wave lithotripsy. J Urol. 1989;142(2 Pt1):263-267.

5. Gaur DD, Trivedi S, Prabhudesai MR, Madhusudhana HR, Gopichand M Laparoscopic ureterolithotomy: technical considerations and long-term followup. BJU Int. 2002;89(4):399-343.

6. Chew BH, Knudsen BE, Denstedt JD. The use of stents in contemporary urology Curr Opin Urol. 2004;14(2):111-115.

7. Soares RS, Romanelli P, Sandoval MA, Salim MM, Tavora JE, Abelha DL Jr Retroperitoneoscopy for treatment of renal and ureteral stones. Int Braz J Urol. 2005;31(2):111-116.

8. Raboy A, Ferzli GS, Ioffreda R, Albert PS. Laparoscopic ureterolithotomy. Urology. 1992;39(3):223-225

9. Wuernschimmel E, Lipsky H. Laparoscopic treatment of an upper ureteral stone. J Laparoendosc Surg. 1993;3(3):301-307.

10. Gangal HT, Gangal PH, Gangal MH. An attempt at a percutaneous retroperitoneoscopic approach to ureterolithotomy. Surg Endosc. 1993;7(5):455-458.

11. Escovar Díaz P, Rey Pacheco M, López Escalante JR, Rodríguez Cordero M, la Riva Rodríguez F, González Zerpa RD, et al. Laparoscopic ureterolithotomy. Arch Esp Urol. 1993;46(7):633-637.

12. Bellman GC, Smith AD. Special considerations in the technique of laparoscopic ureterolithotomy. J Urol. 1994:151(1):146-149.

13. Harewood LM, Webb DR, Pope AJ. Laparoscopic ureterolithotomy, the results of an initial series, and an evaluation of its role in the management of ureteric calculi. Br J Urol. 1994;74(2):170-176.

Correspondencia autor: Dr. Danilo Borges Matias

Servicio de Urología. Hospital Universitario São José

Calle Aimorés, 2896, Santo Agostinho. CEP 30140-073

Belo Horizonte, Minas Gerais, Brasil

Tel.: +55 3132998128

E-mail autor: danilomatiass@gmail.com

Información artículo: Original - Laparoscopia

Trabajo recibido: febrero 2009

Trabajo aceptado: marzo 2009 